\title{
Extremely Low Frequency Electromagnetic Investigation on Mars
}

\author{
Joanna Kozakiewicz ${ }^{1,2}$ (D) Andrzej Kulak ${ }^{3} \cdot$ Jerzy Kubisz $^{1} \cdot$ \\ Krzysztof Zietara
}

Received: 13 May 2016/Accepted: 18 July 2016/Published online: 20 July 2016

(C) The Author(s) 2016. This article is published with open access at Springerlink.com

\begin{abstract}
Natural electromagnetic (EM) signals of extremely low frequencies (ELF, $3 \mathrm{~Hz}-$ $3 \mathrm{kHz}$ ) can be used to study many of the electromagnetic processes and properties occurring in the Martian environment. Sources of these signals, related to electrical activity in the atmosphere, are very significant since they can influence radio wave propagation on the planet, the atmospheric composition, and the ionospheric structure. In addition, such EM signals can be employed in many purposes such as: surveying the subsurface of Mars or studying the impact of the space weather on the Martian ionosphere. As ELF waves propagate on very long distances, it is possible to explore properties of the entire planet using single-station recordings. In this study, we propose an experiment that allows measuring ELF signals from the Martian surface. Such measurements can be used for detection of electric discharges in the atmosphere and water reservoirs in the planetary subsurface.
\end{abstract}

Keywords Mars - Radio waves - Schumann resonance - Electromagnetic exploration

\section{Introduction}

It is possible that some natural EM sources exist on Mars and can be used to investigate the atmospheric electricity, the subsurface structure of the planet, and the properties of the atmospheric layers below the main ionospheric region. This atmospheric group of radio sources is related especially to electric discharges, which can be generated on Mars by

Joanna Kozakiewicz

j.kozakiewicz@uj.edu.pl

Astronomical Observatory, Jagiellonian University, 171 Orla St., 30-244 Kraków, Poland

2 Institute of Geography and Spatial Management, Jagiellonian University, Kraków, Poland

3 Department of Electronics, AGH University of Science and Technology, 30 Mickiewicza St, 30-962 Kraków, Poland 
widespread cloudy phenomena: dust storms and dust devils (Farrell and Desch 2001; Krauss et al. 2006; Zhai et al. 2006).

Once generated, ELF radio waves can propagate on very long distances, many times around the globe, since they are weakly attenuated in planetary environments. They can also penetrate deep into media, since their skin depth is quite large, as compared to waves in higher frequency ranges. ELF wave propagation occurs within a waveguide (a planetary cavity), made of the ground and the ionosphere. When an ELF wave is propagating from its single source, the environmental properties, such as: electrical conductivity of the surface, the subsurface, and the atmosphere, influence its propagation parameters. In addition, when this cavity is excited by a quasi-stationary source of ELF waves, a Schumann resonance (SR) phenomenon arises, owing to a rapid increase of the field amplitude at the natural frequencies of the cavity. The SR parameters as well as the propagation parameters provide information about the global properties of the cavity.

ELF electromagnetic methods are particularly predisposed to examine the parameters of the Martian environment, as due to the low-conductivity surface of the planet (there is no liquid water at the planetary surface), they allow for deep penetration into the Martian ground, and due to low attenuation of these waves, they can be used to detect even relatively low-intensity signals. The electromagnetic sounding in the low frequency range is favorable for detection of deep subsurface water layers, which cannot be done using radar or seismic methods (Delory et al. 2007; Grimm et al. 2009). Using this technique the presence of groundwater at depths up to several kilometers below the surface can be investigated. The advantages of ELF methods, as compared to high-frequency (e.g. radar) techniques, include far greater sensitivity and simpler interpretation of the results (Grimm 2002). The disadvantages are mainly associated with poorer resolution as they typically give only globally averaged results. However, this feature is important in studying global processes and intermittent phenomena.

Therefore, we propose an EM method that allows studying global properties of the Martian cavity as well as individual ELF sources. Such an approach is more robust than the one presented by Grimm et al. (2009), since it allows surveying the atmosphere and the ground on the global scale, and as it is a passive technique, it does not require any antenna deployment system nor additional power supply to generate signals.

\subsection{Measuring Electrical Activity on Mars}

It should be mention that there are several different techniques for detection of electric discharges. Optical measurements are the easiest, however in case of Mars, they are not possible to perform, as dusty phenomena do not allow for such investigation. Also chemical analysis can provide some evidence of discharges (Krasnopolsky 2006). Yet, that approach has also been inconclusive in this case. Other important techniques are related to remote sensing radio observations and in situ measurements.

\subsubsection{Remote Sensing Measurements}

The calculations and observations made by Renno et al. (2003), based on the suggestion that $\mathrm{HF}$ radio emission could be generated by glow discharges (Farrell et al. 1999), led to possible detection of such events on Mars in the microwave range. Renno et al. (2003) concluded that ground-based radio observations, at $22 \mathrm{GHz}$ with the Very Large Array (VLA) telescope, made in 1995, displayed a correlation between high microwave emission and dust devil activity. In 2006, Ruf et al. (2009) also conducted some observation in the 
microwave range using a $34 \mathrm{~m}$ parabolic antenna of the Deep Space Network. They observed unusual pattern of radiation, emitted in minutes-long bursts, when a regional dust storm was present on Mars. They also detected some peaks at approximately $10 \mathrm{~Hz}$, which were explained as detection of Schumann resonance induced by discharges within the dust storm. Anderson et al. (2012) performed similar observation using the Allen Telescope Array in 2010. They found similar variations. However, that variability spread across a broad spectrum and was driven by narrowband radio frequency interferences. The spectral peaks with a periodic $10 \mathrm{~Hz}$ structure were common, appearing one or two per hour, and despite the fact that there was no dust storm on Mars during observations. The authors did not conclude whether observed signals had their source on Mars or on Earth. Also Gurnett et al. (2010) stated that during a 5-year search for radio signals associated with discharges, no signal was discovered although during this period two major and several regional storms occurred. It is worth mentioning that the MARSIS instrument, used in that research, would be capable of the detection of terrestrial lightning activity.

In future, intensive electric discharges on Mars may be detected by large terrestrial radio interferometers such as the Low Frequency ARray (LOFAR) or the Square Kilometer Array (Zarka et al. 2008).

\subsubsection{In Situ, Laboratory, and Analogs' Measurements}

Mills (1977) anticipated that it is necessary to conduct radio observations and electrostatic field measurements at the Martian surface to establish whether electrical discharges are present on Mars. Delory (2010) also stated that a relatively simple experiment to measure radio waves in the spherics band would provide a similar confirmation, as would the detection of Schumann resonance, and that among all possible measurements, observations from instruments on the future in situ network platforms are most likely to produce a significant step in our understanding of the true nature of the atmospheric electricity on Mars.

Although many years ago some experiments and instruments were proposed to study electromagnetic properties of the Martian environment (Berthelier et al. 2000), the only upto-date experimental evidence for electrical activity on Mars is related to electrostatic adhesion of dust to the wheels of rovers (Ferguson et al. 1999; Aplin 2006).

Experiments carried out under laboratory conditions confirmed that agitated dry sand could generate discharges (Eden and Vonnegut 1973; Mills 1977). Krauss et al. (2006) obtained analogous results using a JSC-Mars-1 simulant and glass micro-balloons. On the other hand, the study by Aplin et al. (2012) displayed that electrical discharges occurred only if glass micro-balloons were added into sand particles. Therefore, the Martian simulant may not generate discharges, and the previous studies may be irrelevant as the glass containers, in which the experiments were conducted, affected the observed results by wall-dust interactions.

As there are no measurements on Mars, we can only approximate the electrical activity from Martian dust storms and dust devils by data obtained in the terrestrial environment. Previous studies, carried out especially in Africa, Asia, and North America (Stow 1969; Kamra 1972; Williams et al. 2008; Zhang et al. 2004), showed that electric fields up to hundreds of $\mathrm{kV} / \mathrm{m}$ at the ground level were associated with dust storms. Observations indicated also that dust devils generate: quasi-static DC electric fields of the order of 1-100 kV/m (Freier 1960; Crozier 1964; Farrell et al. 2004; Renno et al. 2004; Zhai et al. 2006), AC currents, and ELF emission (Houser et al. 2003). 
Some information on the electrical activity on Mars will be hopefully provided by the DREAMS payload in the ExoMars 2016 program, in which the MicroARES sensor will be responsible for measurements of electric fields (Deprez et al. 2014). DREAMS will provide new insights into the role of electric forces on dust lifting, and their impact on dust storms initiation.

From all the techniques that allow verifying whether electric discharges are present in the planetary environment, the Schumann resonance investigation is identified as the most informative single measurement and should be a priority when proposing future planetary electrical instrumentation (Berthelier et al. 2000; Aplin et al. 2008; Harrison et al. 2008).

\subsection{Measuring Global Parameters of the Martian Cavity}

The ionosphere of Mars has been investigated by the Mariner (Kliore et al. 1972), the Mars (Vasilev et al. 1975), and the Viking missions (Zhang et al. 1990), the Mars Global Surveyor (Rishbeth and Mendillo 2004; Opgenoorth et al. 2010), the Mars Express (Patzold et al. 2005; Morgan et al. 2008; Gurnett et al. 2008; Nemec et al. 2011; Withers et al. 2012), and the MAVEN (Bougher et al. 2014). The electron density profiles of the Martian atmosphere were obtained by direct measurements, the radio occultation method, and radar sounding. On the basis of these techniques, it is possible to obtain precise models of the atmospheric conductivity. Yet, the ground conductivity profile is still unknown.

On the basis of the measured ELF propagation parameters or the Schumann resonance parameters, the structure of the ground can be estimated by finding inverse solutions of Maxwell's equations in non-uniform mediums. To accomplish such an investigation, an analytical method has been developed (Kulak et al. 2013), and used to investigate the waveguide properties that could be acquired from the impulse waveform measurements and the SR investigation (Kulak et al. 2013; Kozakiewicz et al. 2015). The developed method is computationally efficient. It takes minutes to obtain the results for various environmental models, whereas with numerical modeling it takes days. The potential of the method has been presented in previous publications (Kulak et al. 2013; Kozakiewicz et al. 2015). Using this method, we found the relationship between individual properties of the Martian ground (e.g. the conductivity of the ground layers), and the propagation parameters or the SR parameters (e.g. the phase velocity, the resonant frequency).

When the ground profile is known, it will be possible to investigate the seasonal or temporary changes in the ionosphere using the same technique.

\section{ELF Propagation on Mars}

\subsection{Electric Discharges on Mars}

The importance of the atmospheric electricity can be summarized with the role it plays in chemistry of the atmosphere, aeolian transport, production and destruction of organic compounds. Consequently, it indirectly influences global climate, and presence of life.

Electric discharges are transient events that take place when electric field reaches the breakdown value. The electric field value necessary for the electric breakdown depends on the atmospheric pressure and composition, and can be described by Paschen's law (Raizer 1991): 


$$
E_{b r}=\frac{A p}{B+\ln (p d)}
$$

where $B=\ln [\tilde{A} / \ln (1 / \tilde{\gamma}+1)], B$ and $A$ are constants, depending on the composition, $\tilde{\gamma}$ is the Townsend ionization coefficient, $p$ is the atmospheric pressure, and $d$ is the distance between charges. On Mars, due to low atmospheric pressure, the electric breakdown (for $d=1 \mathrm{~m}$ ) is ca. $20-25 \mathrm{kV} / \mathrm{m}$, whereas on Earth, it is ca. $3 \mathrm{MV} / \mathrm{m}$ at sea level (Melnik and Parrot 1998).

In an idealized model, charge generation in dusty phenomena is related to triboelectricity among sand particles and the ground, and between sand particles and dust particles. During a dust phenomenon, smaller particles, which are negatively charged, are carried by wind to higher altitudes, whereas heavier grains are concentrated near the ground. This leads to charge separation.

The electric breakdown increases with decreasing distance between electrodes [see (1)]. In the basic mode of sand transport, called saltation, this value, related to the distance between two populations of grains, is ca. $30 \mathrm{~cm}$, which leads to a higher value of the electric breakdown (Kok and Renno 2009). In addition, in saltation, electric fields exceeding $5 \mathrm{kV} / \mathrm{m}$ accelerate free electrons to energies sufficient to ionize $\mathrm{CO}_{2}$ molecules (Delory et al. 2006). The increase in ion and electron concentrations enhances the atmospheric conductivity, neutralizing the charges and limiting further increase in the electric field. This negative feedback limits the electric field to $15-20 \mathrm{kV} / \mathrm{m}$ and rules out the initiation of electric discharges (Kok and Renno 2009). Yet these findings do not limit discharges in dust devils and dust storms, where electric discharges are associated not solely with saltation but also suspension, and in which charge separation is not limited to small distances.

For comparative purposes, we can assume that on Mars, there are vertical electric discharges characterized by the current given by (Jones 1970; Cummer and Farrell 1999): $I(t)=I_{0}\left(e^{-t / \tau_{\alpha}}-e^{-t / \tau_{\beta}}\right)$, where $I_{0}, \tau_{\alpha}$, and $\tau_{\beta}$ are constants, and similar to terrestrial values: $I_{0} \sim 20 \mathrm{kA}, \tau_{\alpha} \sim 50 \mu \mathrm{s}$, and $\tau_{\beta} \sim 5 \mu$ s (Cummer and Farrell 1999), implying that the stroke duration, $\tau=\tau_{\alpha}-\tau_{\beta}$, is ca. $45 \mu$ s. This source generates a peak current ca. $14 \mathrm{kA}$.

We may assume that the current propagates with exponentially decreasing velocity $v$, given by: $v(t)=v_{0} e^{-t / \tau_{\lambda}}$, where $\tau_{\gamma} \sim 33 \mu \mathrm{s}$, and $v_{0}=8 \times 10^{7} \mathrm{~m} / \mathrm{s}$ (Cummer 1997). Therefore, the total length of the discharge channel is: $\ell_{0}=\int_{0}^{\infty} v(t) d t$, which gives $=2.6 \mathrm{~km}$, and the channel length can be given by: $\ell(t)=\ell_{0}\left(1-e^{-t / \tau_{\gamma}}\right)$. Hence the current moment $s(t)$, can be calculated as:

$$
s(t)=I(t) \ell(t)=I_{0} \ell_{0}\left(e^{-t / \tau_{\alpha}}-e^{-t / \tau_{\beta}}\right)\left(1-e^{-t / \tau_{\gamma}}\right) .
$$

By the Fourier transform of (2), we get the spectral source current moment $s(f)$. For low frequencies (below $3 \mathrm{kHz}$ ), the spectrum is almost flat and equals to the electric dipole moment (charge moment) of the return stroke, which can be defined as (Kulak et al. 2010):

$$
\tilde{p}=\int_{0}^{\infty} s(t) d t=I_{o} \ell_{0}\left(\tau_{\alpha}-\tau_{\beta}-\frac{\tau_{\alpha} \tau_{\gamma}}{\tau_{\alpha}+\tau_{\gamma}}+\frac{\tau_{\beta} \tau_{\gamma}}{\tau_{\beta}+\tau_{\gamma}}\right) .
$$

This means that the return stroke can be described in the ELF range as a very short impulse of amplitude given by (3). Using (3), we get that the studied discharge is 
characterized by a charge moment: ca. $1 \mathrm{C} \mathrm{km}$. On Earth, a typical discharge has a charge moment ca. 6.2 C km (Kulak et al. 2010).

The waveforms related to the studied Martian discharge, as observed at the $5 \mathrm{Mm}$ distance, were calculated for different models of the Martian waveguide in the work of Kulak et al. (2013).

\subsection{Theoretical Aspects of the Martian Waveguide}

We can calculate the amplitude of the magnetic field component in the Martian cavity, using the analytical method. The maximum amplitude spectral density of the magnetic field component in the Martian cavity can be described as (Mushtak and Williams 2002):

$$
H_{\varphi}(\vartheta, f)=-\frac{s(f)}{4 h_{m} R} \frac{P_{\bar{v}}^{1}[\cos (\pi-\vartheta)]}{\sin \left[\pi \bar{v}_{S}(f)\right]},
$$

where $P_{\bar{v}}^{1}$ is the Legendre function of complex order, $h_{m}$ is the real part of the magnetic altitude (the altitude that describes the distance between currents generated by the magnetic field component within the boundary mediums), $s(f)$ is the spectral current moment of the source, $\theta$ is the distance from the source, $\bar{v}_{S}$ is the complex propagation parameter for a spherical cavity.

As the subsurface layers are unknown values of the Martian environment, we have investigated the influence of various hypothetical ground models on ELF propagation. Particularly, we considered two important situations: the ground with some brines (Brine model), and the ground without water (Dry model). In both models, the upper layer of the ground was of low conductivity $\left(\leq 10^{-7} \mathrm{~S} / \mathrm{m}\right)$, and the lower layers were of higher conductivity. In the Brine model, these layers' conductivity was as high as $0.01 \mathrm{~S} / \mathrm{m}$, and they were located 1-2 km below the surface. In the Dry model, the higher conductivity layers (ca. $10^{-4} \mathrm{~S} / \mathrm{m}$ ) were located at the depth of $40 \mathrm{~km}$ (Kozakiewicz et al. 2015).

The exact formulae for the magnetic altitude for the ground models were given in the work of Kozakiewicz et al. (2015). For a single source that had the form of the Dirac delta function and amplitude $1 \mathrm{C} \mathrm{km}$, the results indicated that the measured magnetic amplitude, at the distance, at which we obtained the best visibility of the Schumann resonance first mode, was $3.3 \times 10^{-4} \mathrm{pT} / \sqrt{\mathrm{Hz}}$ for the Dry model, and $4.6 \times 10^{-4} \mathrm{pT} / \sqrt{\mathrm{Hz}}$ for the Brine model.

On the other hand, the minimum fluctuation of spectral intensity, $\Delta\left|B_{s}\right|^{2}$, that the magnetic antenna enables to detect can be given by (Kulak et al. 2014):

$$
\Delta\left|B_{s}\right|^{2}=\left|B_{n}\right|^{2} \frac{1}{\sqrt{\Delta f \tau}}
$$

where $\left|B_{n}\right|^{2}$ is the spectral density of the magnetic antenna self-noise, $\Delta f$ is the frequency range, and $\tau$ is the recording time. The power spectrum of the Schumann resonance signal on Mars can be characterized by (Nickolaenko and Hayakawa 2002):

$$
|B(f)|^{2} \sim \lambda_{p}\left\langle\tilde{p}^{2}\right\rangle
$$

where $\lambda_{p}$ is the discharge rate, $\tilde{p}$ is given by (3).

The cutoff frequency of the Martian waveguide for the Dry and the Brine model, given as $c /(2 h)$, where $c$-is the speed of light in free space, and $h-$ is the distance between the 
waveguide boundaries (ca. $135 \mathrm{~km}$ in the Dry model, and ca. $100 \mathrm{~km}$ in the Brine model), is 1.1 , and $1.5 \mathrm{kHz}$, respectively.

\section{ELF Experiment on Mars}

\subsection{Description of the Measuring System}

As the considered phenomena are mainly of very low intensity, we propose a high-sensitivity and low-noise system for measurements on Mars. The receiver bandwidth is proposed to be $30 \mathrm{mHz}$ to $1.5 \mathrm{kHz}$ to cover also the cutoff frequency region. The continuous raw data will be recorded to allow spectral and waveform measurements. As electromagnetic instruments placed near the system may interfere with its measurements, it is necessary to consider it as an autonomous platform.

The sounding system consists of two orthogonal horizontal magnetic antennas, with built-in preamplifiers, an active vertical electric antenna, and a receiver. The receiver has the basic functions of auto-calibration and self-diagnosis. It is also possible to disconnect power from the unused (or damaged) measuring circuits. The electric antenna is $400 \mathrm{~mm}$ long. This short electric dipole is a wire ended at the top with a metal sphere. Each magnetic antenna is $700 \mathrm{~mm}$ long and is $10 \mathrm{~mm}$ in diameter. Their design is based on a wire-wound induction coil with a core of high-permeability material.

The magnetic antennas were selected in a way to maximize the signal-to-noise ratio and minimize their weight and size. The optimization method was described in a work of Kulak (2010), and on the basis of this method several generations of ELF antennas have been built (Kulak et al. 2014). The active magnetic antenna sensitivity depends mainly on: the length $l_{a}$, and the diameter $d_{a}$ of the antenna core, as well as the noise factor of the antenna amplifier, $F_{a}$. The mass of the antenna is, almost exclusively, equal to the mass of the core and induction coil; the mass of the electric circuitry is negligible. The antenna masssensitivity optimization leads to establishing a ratio between the mass of the core and the mass of the coil, and therefore, to defining the outer diameter of the antenna. Using an iron core, and an aluminum coil, the outer diameter can be approximated by $3 d_{a}$, and the antenna noise for a unit bandwidth $B_{n}$ can be given by (Kulak 2010):

$$
B_{n}=0.028 \frac{\sqrt{F_{a}-1}}{d_{a}^{0.3} l_{a}^{2.2} f}
$$

where $f$ is the operating frequency. From (6), and for the amplifier with the noise factor equals to 1.6 (at $10 \mathrm{~Hz}$ ), the antenna of the size proposed above has a noise level ca. $0.02 \mathrm{pT} / \sqrt{\mathrm{Hz}}$.

From (4) and (5), with $\Delta f=1 \mathrm{~Hz}, \tau_{R}=300 \mathrm{~s}$, and the magnetic component of the first SR frequency, presented in point 2.2, we find that the platform should easily detect Schumann resonance even for the Dry model if the intensity of discharges on the planet, described by $\lambda_{p}\left\langle\tilde{p}^{2}\right\rangle$, is of order of $10^{2} \mathrm{C}^{2} \mathrm{~km}^{2} / \mathrm{s}$. On Earth, the discharge intensity is of order of $10^{3} \mathrm{C}^{2} \mathrm{~km}^{2} / \mathrm{s}$.

Weaker sources can be detected with the electric antenna. However, this antenna does not allow estimating the location of the source. Using an amplifier characterized by small input capacitance (in comparison to the self-capacitance of the antenna), high impendence $\sim 10^{9} \Omega$, and with the input noise level $U_{n}=2 \mathrm{nV} / \sqrt{\mathrm{Hz}}$ at $10 \mathrm{~Hz}$, we can calculate that 
the antenna enables to detect electric fields of intensity $E_{n}$, given by (Nickolaenko and Hayakawa 2014):

$$
E_{n}=\frac{U_{n}}{h_{\text {eff }}},
$$

where $h_{\text {eff }}$ is the effective height of the antenna. From (7), assuming that $h_{\text {eff }} \approx$ $h_{a}=400 \mathrm{~mm}$, the detectable electric field limit is $5 \mathrm{nV} / \mathrm{m} / \sqrt{\mathrm{Hz}}$. The electric antenna noise for the studied bandwidth is ca. $200 \mathrm{nV} / \mathrm{m}$. This antenna enables to study a single discharge described in point 2.1, as its peak ELF amplitude should not be lower than $E_{\mathrm{p}}=\mathrm{c} \times B_{\mathrm{p}} \sim 10^{3} \mathrm{nV} / \mathrm{m}$ at the $5 \mathrm{Mm}$ distance (Kulak et al. 2013; Cummer and Farrell 1999).

\subsection{Description of the Martian Platform}

Apart from the scientific payload the platform consists of a general processing unit (GPU), communication unit (CU), with a UHF antenna, power management unit (PMU), solar panel (SP), and a lithium-ion battery (BAT). The block diagram of the scientific payload within the platform is presented in Fig. 1, and the conceptual 3D visualization of the station in Fig. 2.

The magnetic antennas are placed in cylindrical hermetic boxes. They are connected to multi-channel receiver unit, which consists of analog circuitry and analog to digital converters (ADCs). Digitized data from the converters are received and processed by the data acquisition unit (DAU) in the GPU module. The role of the analog circuitry, containing amplifiers, and antialiasing filters, is to strengthen and reduce the bandwidth of the measured signals, before they reach synchronous ADCs. It is hard to establish the dynamic range of ELF fields on Mars, though probably 16-bit ADCs are sufficient to achieve suitable measurements. The receivers' inputs are equipped with switches which allow

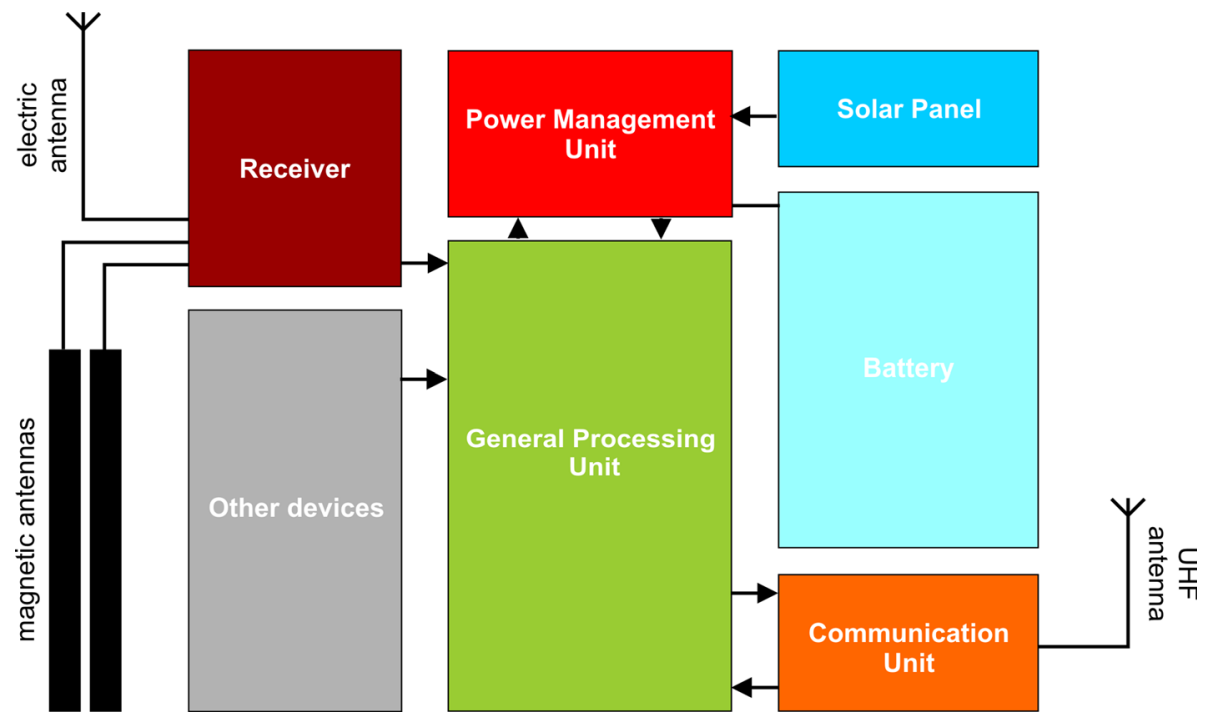

Fig. 1 Block diagram of the ELF station 


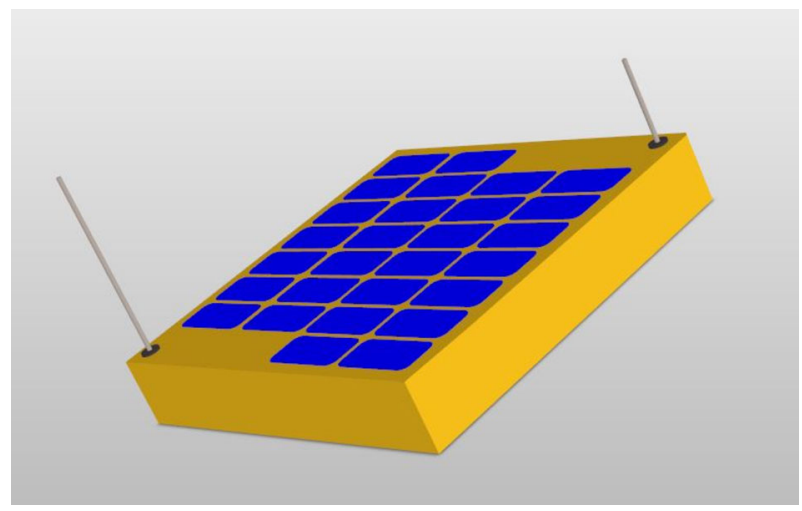

Fig. $23 \mathrm{D}$ visualization of the platform

antenna's cross switching. This improves calibration accuracy, increases reliability and enables additional tests and measurements after landing on Mars.

The GPU controls all the units of the platform. It is used to implement all the tasks connected with data acquisition from the receiver's ADC's, data preprocessing, preanalysis, compression, buffering and system timing. During normal operation GPU performs scheduled measurement runs, collects status reports from individual modules, monitors health and ambient parameters of the station, and periodically sends status information to Earth. In case of technical problems or lack of communication with Earth, GPU performs special scenarios. The GPU is equipped with memory of adequate capacity, which is crucial for data buffering during communication.

The primary function of the communication unit is to provide a radio connection with a Martian orbiter, testing the current link quality, and adjusting transmission power. Although it is possible to use direct communication with Earth, the uplink to orbiters allows sending much more data. Therefore, the surface-to-orbit communication is considered to be more convenient than the direct Earth link. However, even using the surfaceto-orbit communication, it is not possible to send all raw data during one sol. Therefore, at first only some processed information will be send, and then, only if there are any interesting features in the recordings, the rest of the data will be uploaded. Hence the on-board memory necessary for buffering data is quite large, ca. 2 GB.

The main role of the power management unit is to control the energy budget, especially for the GPU. In case of emergency, it can cut off energy supply to other modules. The main tasks of the PMU are supervision of the battery and the solar panels, forecasting time at a given load, and handling emergency situations.

The constraints on power supply and temperature were decisive factors to locate the platform in a tropical region. The temperature at the surface can vary significantly during the Martian sol. In cold Martian conditions, the electronics must be well protected. In this design, the platform is equipped with the external and internal electronic warm boxes. The external electronic warm box (EEWB) has dimensions $850 \mathrm{~mm} \times 850 \mathrm{~mm} \times 150 \mathrm{~mm}$. In the EEWB are placed the magnetic antennas and the internal electronic warm box (IEWB). On the EEWB surface are mounted both the electric and UHF antennas. The EEWB is made of a composite thermally insulated with aerogel, and its wall is $20 \mathrm{~mm}$ thick. Its interior dimensions are $810 \mathrm{~mm} \times 810 \mathrm{~mm} \times 110 \mathrm{~mm}$. The IEWB is made of the same composite as the EEWB. It provides a support structure for the entire electronics and 
additional thermal insulation. Its internal dimensions are $625 \mathrm{~mm} \times 625 \mathrm{~mm} \times 80 \mathrm{~mm}$. The heat generated by the electronics is sufficient to support suitable operation of the station, and due to the fact that only small amount of heat is produced a heat rejection system is not necessary.

The whole station including all units requires not more than $120 \mathrm{Wh}$ per sol. The solar panel covers almost the whole surface of the EEWB and should provide minimum $350 \mathrm{Wh}$ per sol. As within the platform is enough space, and the amount of energy provided by the panels is larger than necessary, other measuring instruments, characterized by low electromagnetic noise can be located within the platform to extend scientific payload.

All instruments inside the EEWB are insulated from sand, dust, and UV emission. Only the electric antenna is affected by both photoemission and impacts of charged dust particles. The mass of the platform, ca. $8 \mathrm{~kg}$, enables steady operation even during strong gusts of wind. As the station must be placed in an appropriate position, it is considered to be a deployable part of a rover or a lander.

\section{Discussion}

To study electromagnetic properties of a planet, passive and active electromagnetic exploration can be employed. The passive sensors can be used when natural EM sources are present in the planet, the active when those sources are of insufficient intensity.

It is not possible to locate any ELF transmitter at the Martian surface due to large dimensions and high power consumption. But it is possible to employ natural sources of electromagnetic waves in the ELF and ULF (Ultra Low Frequency, $3 \mathrm{mHz}-3 \mathrm{~Hz}$ ) range. These include electric discharges as well as emission generated by interactions among the solar wind, magnetosphere, ionosphere and the crustal magnetization. The risk related with the assumption of the existence of those natural sources on Mars is probably smaller than the one connected with finding of life. In addition, lack of detection of any of those sources during the experiment also carries important information on the Martian environment.

The proposed Mars station enables detecting beside ELF also ULF signals, similarly like our ELF station on Earth, which has been measuring the magnetic field components since 1994 (Kulak et al. 2014). Research projects based on the data obtained from this station comprise studying the signals related to fields generated in the magnetosphere, lower atmosphere and ionosphere. Yet, the magnetic sources on Mars cannot be identified by any simple and direct analogy with the terrestrial phenomena due to the complex nature of the magnetosphere of Mars. The most likely and continual sources of magnetic origin are probably related to Sq current system and magnetic pulsations (Grimm 2002; Fillingim et al. 2010). Yet, only the latter would be able to generate signals in the ULF-ELF range. Time-varying solar wind perturbations of the mini-magnetospheres, changeable ionospheric currents, and auroras might produce useful signals. However, our insufficient knowledge of electromagnetic processes in the Martian ionosphere and magnetosphere, does not allow precise determination of their properties and the possibility of their measurements from the planetary surface.

Although a single station provides enough data for basic research, as the magnetic antennas allow calculating the direction of a wave arrival, a single station is not sufficient to determine a precise location of individual discharges or groundwater reservoirs. The network of platforms is, therefore, favorable. Such a network may consist of three or four stations located at different parts of Mars, with a baseline larger than $1 \mathrm{Mm}$. 


\section{Conclusions}

An ELF station located at the Martian surface enables studying Mars from the within but still on the global scale. As such experiment is relatively simple, it should be considered for studying the Martian environment during the future in situ missions.

Using the analytical approach, it is possible to establish the influence of various environmental properties on the SR parameters or the ELF propagation parameters. Knowing these relationships, it should be possible to estimate the approximated properties of the Martian environment. Yet, Mars has shown repeatedly that our state of knowledge in planetary science is not as advanced as we would like. As we measure the described phenomena solely on Earth, on Mars, some physical processes or environmental properties, which we have not predicted nor expected, may affect the results obtained from the ELF station measurements. However, any observed ELF signal in the Martian cavity will indicate the existence of ELF sources. In addition, if the measured parameters are much lower than those estimated on the basis of the exact profiles of the atmospheric conductivity, then we would be able to state that there is no liquid water in the subsurface down to several kilometers.

Acknowledgments This work has been supported by the National Science Centre Grants 2012/04/M/ST10/ 00565 and 2013/09/N/ST9/02200.

Open Access This article is distributed under the terms of the Creative Commons Attribution 4.0 International License (http://creativecommons.org/licenses/by/4.0/), which permits unrestricted use, distribution, and reproduction in any medium, provided you give appropriate credit to the original author(s) and the source, provide a link to the Creative Commons license, and indicate if changes were made.

\section{References}

M.M. Anderson, A.P.V. Siemion, W.C. Barrot, G.C. Bower, G.T. Delory, I. De Pater, D. Werthimer, The allen telescope array search for electrostatic discharges on mars. Astrophsical J. 744(15), 1-13 (2012)

K.L. Aplin, Atmospheric electrification in the solar system. Surv. Geophys. 27, 63-108 (2006)

K.L. Aplin, R.G. Harrison, M.J. Rycroft, Investigating earth's atmospheric electricity: a role model for planetary studies. Space Sci. Rev. 137, 11-27 (2008)

K.L. Aplin, T. Goodman, K.L. Herpoldt, C.J. Davis, Laboratory analogues of Martian electrostatic discharges. Planet. Space Sci. 69, 100-104 (2012)

J.J. Berthelier, R. Grard, H. Laakso, M. Parrot, ARES, atmospheric relaxation and electric field sensor, the electric field experiment on NETLANDER. Planet. Space Sci. 48, 1193-1200 (2000). doi:10.1016/ S0032-0633(00)00103-3

S.W. Bougher, T.E. Cravens, J. Grebowsky, J. Luhmann, The aeronomy of Mars: characterization by MAVEN of the upper atmosphere reservoir that regulates volatile escape. Space Sci. Rev. (2014). doi:10.1007/s11214-014-0053-7

W.D. Crozier, The electric field of a New Mexico dust devil. J. Geophys. Res. 69, 5427-5429 (1964)

S.A. Cummer, Lightning and ionospheric remote sensing using VLF/ELF radio atmospherics. PH.D. Thesis, Stanford University, pp. 137 (1997)

S.A. Cummer, W.M. Farrell, Radio atmospheric propagation on Mars and potential remote sensing application. J. Geophys. Res. 104(E6), 14149-14157 (1999). doi:10.1029/1998JE000622

G. Delory et al., Oxidant enhancement in martian dust devils and storms: storm electric fields and electron dissociative attachment. Astrobiology 6(3), 451-462 (2006)

G.T. Delory, Electrical phenomena on the Moon and Mars. in Proceedings of ESA Annual Meeting on Electrostatics, Paper A1 (2010)

G.T. Delory, R.E. Grimm, T. Nielsen, W.M. Farrell, Prospecting for subsurface liquid water using magnetotellurics on Mars. LPI Contrib. 1353, 3293 (2007) 
G. Deprez, F. Montmessin, O. Witasse, L. Lapauw, F. Vivat, S. Abbaki, D. Moirin, R. Trautner, R. HassenKhodja, E. d'Almeida, L. Charnenal, J.-J. Berthelier, F. Esposito, S. Debei, S. Rafkin, E. Barth, P. Granier, Micro-ARES, an electric-field sensor for exomars 2016: ealectric fields modelling, sensitivity evaluations and end-to-end tests. LPI Contrib. 1791, 1290 (2014)

H.F. Eden, B. Vonnegut, Electrical breakdown caused by dust motion in low-pressure atmosphere: consideration of Mars. Science 180, 962-963 (1973)

W.M. Farrell, M.D. Desch, Is there a Martian atmospheric electric circuit? J. Geophys. Res. (2001). doi:10. 1029/2000JE001271

W.M. Farrell et al., Electric and magnetic signatures of dust devils from the 2000-2001 MATADOR desert tests. J. Geophys. Res. 109, E03004 (2004). doi:10.1029/2003JE002088

W.M. Farrell, M.L. Kaiser, M.D. Desch, J.G. Houser, S.A. Cummer, Detecting electrical activity from Martian dust storms. J. Geophys. Res. 104(2), 3795-3801 (1999)

D.C. Ferguson, J.C. Kolecki, M.W. Siebert, D.M. Wilt, J.R. Matijevic, Evidence for Martian electrostatic charging and abrasive wheel wear from the Wheel Abrasion Experiment on the Pathfinder Sojourner rover. J. Geophys. Res. 104(E4), 8747-8759 (1999). doi:10.1029/98JE02249

M.O. Fillingim, L.M. Peticolas, R.J. Lillis, D.A. Brain, J.S. Halekas, D. Lummerzheim, S.W. Bougher, Localized ionization patches in the nighttime ionosphere of Mars and their electrodynamic consequences. Icarus 206(1), 112-119 (2010)

G.D. Freier, The electric field of a large dust devil. J. Geophys. Res. 65, 3504 (1960)

R.E. Grimm, Low-frequency electromagnetic exploration for groundwater on Mars. J. Geophys. Res. (2002). doi:10.1029/2001JE001504

R.E. Grimm, Barry Berdanier, Robert Warden, James Harrer, Raymond Demara, James Pfeiffer, Richard Blohm, A time-domain electromagnetic sounder for detection and characterization of groundwater on Mars. Planet. Space Sci. 57(11), 1268-1281 (2009)

D.A. Gurnett, R.L. Huff, D.D. Morgan, A.M. Persoon, T.F. Averkamp, D.L. Kirchner, F. Duru, F. Akalin, A.J. Kopf, E. Nielsen, A. Safaeinili, J.J. Plaut, G. Picardi, An overview of radar soundings of the martian ionosphere from the Mars Express spacecraft. Adv. Space Res. 41(9), 1335-1346 (2008)

D.A. Gurnett, D.D. Morgan, L.J. Granroth, B.A. Cantor, W.M. Farrell, J.R. Espley, Non-detection of impulsive radio signals from lightning in Martian dust storms using the radar receiver on the Mars Express spacecraft. Geophys. Res. Lett. (2010). doi:10.1029/2010GL044368

R.G. Harrison, K.L. Aplin, F. Leblanc, Y. Yair, Planetary atmospheric electricity. Space Sci. Rev. 137, 5-10 (2008)

J.G. Houser, W.M. Farrell, S.M. Metzger, ULF and ELF magnetic activity from a terrestrial dust devil. Geophys. Res. Lett. 30, 1027 (2003). doi:10.1029/2001GL014144

D.L. Jones, Electromagnetic radiation from multiple return strokes of lightning. Atmos. Terr. Phys. 32, 1077 (1970)

A.K. Kamra, Measurements of the electrical properties of dust storms. J. Geophys. Res. 77, 5856-5869 (1972)

A.J. Kliore, D.L. Cain, G. Fjeldbo, B.L. Seidel, M.J. Sykes, S.I. Rasool, The atmosphere of Mars from Mariner 9 radio occultation measurements. Icarus 17, 484-516 (1972)

J.F. Kok, N.O. Renno, Electrification of wind-blown sand on Mars and its implications for atmospheric chemistry. Geophys. Res. Lett. 36, L05202 (2009). doi:10.1029/2008GL036691

J. Kozakiewicz, A. Kulak, J. Mlynarczyk, Analytical modeling of Schumann resonance and ELF propagation parameters on Mars with a multi-layered ground. Planet. Space Sci. 117, 127-135 (2015). doi:10.1016/j.pss.2015.05.019

V.A. Krasnopolsky, A sensitive search for nitric oxide in the lower atmospheres of Venus and Mars: detection on Venus and upper limit for Mars. Icarus 182, 80-91 (2006)

C.E. Krauss, M. Horanyi, S. Robertson, Modeling the formation of electrostatic discharges on Mars. J. Geophys. Res. 111, E02001 (2006). doi:10.1029/2004JE002313

A. Kulak, Czujniki i system do pomiaru naturalnychpól elektromagnetycznych ekstremalnie niskiej częstotliwości na powierzchni Ziemi. Elektronika 01, 106-113 (2010)

A. Kułak, Z. Nieckarz, S. Zięba, Analytical description of ELF transients produced by cloud-to-ground lightning discharges. J. Geophys. Res. 115, D19104 (2010). doi:10.1029/2009JD013033

A. Kulak, J. Kubisz, S. Klucjasz, A. Michalec, J. Mlynarczyk, Z. Nieckarz, M. Ostrowski, S. Zieba, Extremely low frequency electromagnetic field measurements at the Hylaty station and methodology of signal analysis. Radio Sci. 49, 361-370 (2014). doi:10.1002/2014RS005400

A. Kulak, J. Mlynarczyk, J. Kozakiewicz, An analytical model of ELF radiowave propagation in groundionosphere waveguides with a multilayered ground. IEEE Trans. Antennas Propag. (2013). doi:10. 1109/TAP.2013.2268244 
O. Melnik, M. Parrot, Electrostatic discharge in Martian dust storms. J. Geophys. Res. 103(A12), 29107-29117 (1998)

A.A. Mills, Dust clouds and frictional generation of glow discharges on Mars. Nature 268, 614 (1977)

D.D. Morgan, D.A. Gurnett, D.L. Kirchner, J.L. Fox, E. Nielsen, J.J. Plaut, Variation of the Martian ionospheric electron density from Mars Express radar soundings. J. Geophys. Res. 113, A09303 (2008). doi:10.1029/2008JA013313

V.C. Mushtak, E.R. Williams, ELF propagation parameters for uniform models of the Earth-ionosphere waveguide. J. Atmos. Sol. Terr. Phys. 64, 1989-2001 (2002). doi:10.1016/S1364-6826(02)00222-5

F. Nemec, D.D. Morgan, D.A. Gurnett, F. Duru, V. Truhlík, Dayside ionosphere of Mars: empirical model based on data from the MARSIS instrument. J. Geophys. Res. 116, E07003 (2011). doi:10.1029/ 2010JE003789

A.P. Nickolaenko, M. Hayakawa, Resonances in the Earth-Ionosphere Cavity (Kluwer, Dordrecht, 2002), p. 380

A.P. Nickolaenko, M. Hayakawa, Schumann Resonance for Tyros (Springer, Heidelberg, 2014), p. 348

H.J. Opgenoorth, R.S. Dhillon, L. Rosenqvist, M. Lester, N.J.T. Edberg, S.E. Milan, P. Withers, D. Brain, Day-side ionospheric conductivities at Mars. Planet. Space Sci. 58(10), 1139-1151 (2010)

M. Patzold, S. Tellmann, B. Hausler, D. Hinson, R. Schaa, G.L. Tyler, A sporadic third layer in the ionosphere of Mars. Science 310, 837-839 (2005). doi:10.1126/science.1117755

Y.P. Raizer, Gas Discharge Physics (Springer, Heidelberg, 1991), p. 449

N.O. Renno, A.-S. Wong, S.K. Atreya, I. de Pater, M. Roos-Serote, Electrical discharges and broadband radio emission by Martian dust devils and dust storms. Geophys. Res. Lett. 30(22), 2140 (2003). doi:10.1029/2003GL017879

N.O. Renno et al., MATADOR 2002: a pilot field experiment on convective plumes and dust devils. J. Geophys. Res. 109, E07001 (2004). doi:10.1029/2003JE002219

H. Rishbeth, M. Mendillo, Ionospheric layers of mars and earth. Planet. Space Sci. 52, 849-852 (2004). doi:10.1016/j.pss.2004.02.007

C. Ruf, N.O. Renno, J.F. Kok, E. Bandelier, M.J. Sander, S. Gross, L. Skjerve, B. Cantor, Emission of nonthermal microwave radiation by a Martian dust storm. Geophys. Res. Lett. 36, L13202 (2009). doi:10. 1029/2009GL038715

C.D. Stow, Dust and sand storm electrification. Weather 24, 134-140 (1969)

M.B. Vasilev, A.S. Vyshlov, M.A. Kolosov, N.A. Savich, V.A. Samovol, L.N. Samoznaev, A.I. Sidorenko, IuN Aleksandrov, A.I. Danilenko, V.M. Dubrovin, Preliminary results of the two-frequency radiotransillumination of the Martian ionosphere by means of the "Mars" automatic interplanetary stations in 1974. Cosmic Res. 13, 41-45 (1975)

E. Williams, N. Nathou, E. Hicks, C. Pontikis, B. Russell, M. Miller, M.J. Bartholomew, The electrification of dust-lofting gust fronts ('haboobs') in the Sahel. Atmos. Res. 91(2), 292-298 (2008)

P. Withers et al., A clear view of the multifaceted dayside ionosphere of Mars. Geophys. Res. Lett. 39, L18202 (2012). doi:10.1029/2012GL053193

P. Zarka, W. Farrell, G. Fischer, A. Konovalenko, Ground-based and space-based radio observations of planetary lightning. Space Sci. Rev. 137, 257-269 (2008)

Y. Zhai, S.A. Cummer, W.M. Farrell, Quasi-electrostatic field analysis and simulation of Martian and terrestrial dust devils. J. Geophys. Res. 111, E6 (2006). doi:10.1029/2005JE002618

H.-F. Zhang, T. Wang, J.-J. Qu, M.-H. Yan, An experimental and observational study on the electric effect of sandstorms. Chin. J. Geophys. 47(1), 53-60 (2004)

M.H.G. Zhang, J.G. Luhmann, A.J. Kliore, J. Kim, A post-pioneer Venus reassessment of the Martian dayside ionosphere as observed by radio occultation methods. J. Geophys. Res. 95, 14829-14839 (1990). doi:10.1029/JB095iB09p14829 